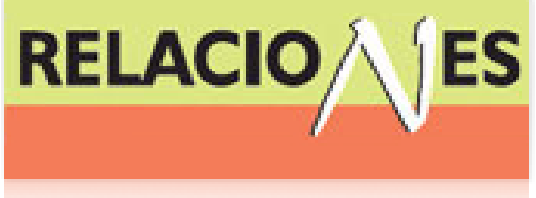

Relaciones. Estudios de historia y sociedad ISSN: 0185-3929

relacion@colmich.edu.mx

El Colegio de Michoacán, A.C

México

Yáñez Andrade, Juan Carlos

Tratado de extradición y protección contra el anarquismo (1901-1902)

Relaciones. Estudios de historia y sociedad, vol. XXXII, núm. 125, 2011, pp. 125-136

El Colegio de Michoacán, A.C

Zamora, México

Disponible en: http://www.redalyc.org/articulo.oa?id=13718526005

Cómo citar el artículo

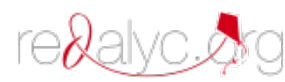

- Número completo

- Más información del artículo

Página de la revista en redalyc.org

Sistema de Información Científica

Red de Revistas Científicas de América Latina, el Caribe, España y Portugal Proyecto académico sin fines de lucro, desarrollado bajo la iniciativa de acceso abierto 


\title{
Tratado de extradición y protección contra el anarquismo (1901-1902) ${ }^{1}$
}

\author{
Juan Carlos Yáñez Andrade*
}

EHESS- PARIS. BECARIO CONICYT, CHILE

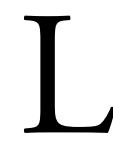

a necesidad de estrechar lazos comerciales de Estados Unidos con los países latinoamericanos forzó al gobierno estadounidense a promover una política de acercamiento y colaboración regional que se denominó panamericanismo. De manera temprana, el presidente Chester Arthur (1881-1885) promovió misiones a los países del continente con el fin concreto de avanzar en una alianza comercial. ${ }^{2}$ Posteriormente, el secretario de Estado, James Blaine, logró del Congreso la autorización para la convocatoria de la Primera Conferencia Panamericana. Ésta fue inaugurada en Washington el 1 de octubre de 1889. Entre los temas tratados estuvieron el establecimiento de una unión aduanera, una moneda común para las transacciones comerciales, la adopción de un sistema uniforme de pesos y medidas y la formulación de un plan de arbitraje para las disputas interamericanas. ${ }^{3}$

La Oficina de las Repúblicas Americanas -que después se conocería como Unión Panamericana- fue creada para funcionar como órgano ejecutivo permanente, con funciones iniciales de coordina-

*yanezandrade@gmail.com

${ }^{1}$ Actas y documentos. Segunda Conferencia Pan-Americana, México, Tipografía de la Oficina Impresora de Estampillas, 1902.

${ }^{2}$ Ernesto Quesada, La evolución del panamericanismo, Buenos Aires, Talleres Gráficos del Ministerio de Agricultura de la Nación, 1919, 40.

${ }^{3}$ Miguel Ángel Fernández, "I Conferencia Panamericana (Washington, 1889-1890). Prolegómenos para un Derecho Internacional Americano", en Carlos Marichal, México y las Conferencias Panamericanas, 1889-1938, México, Secretaría de Relaciones Exteriores, 2002. 
ción comercial. Su sede estaba en Washington y bajo la supervisión del Departamento de Estado, al ser su presidente el secretario de Estado de ese país. Su Consejo Ejecutivo estaba formado por los representantes diplomáticos acreditados ante la Casa Blanca. Pronto se le fueron agregando a la Oficina agencias encargadas de asesorar y realizar investigaciones sobre distintas materias. En perspectiva, las conferencias panamericanas pronto superarían la discusión puramente comercial para avanzar en un conjunto de materias, muchas de las cuales eran parte de la agenda internacional que le interesaba promover al gobierno de los Estados Unidos. A partir de la Segunda Conferencia se avanzaría en políticas culturales, con la creación de un catálogo de obras americanas, el canje de publicaciones entre los países y la creación de una biblioteca especializada en temáticas de integración regional. También se fomentarían las comunicaciones del continente, promoviendo la construcción de un ferrocarril y de una carretera panamericana. Por último, en políticas de salud y seguridad social se avanzaría en la creación de una Oficina Panamericana de la Salud.

En cuanto al documento que analizamos, éste fue discutido en la Segunda Conferencia, realizada en la ciudad de México entre diciembre de 1901 y enero de 1902. Aquí se acordó coordinar los esfuerzos de lucha en contra de los movimientos anarquistas del continente. El anarquismo había hecho su entrada en América Latina a fines del siglo XIx junto con la llegada de corrientes de inmigrantes europeos en búsqueda de mejores condiciones de trabajo. Por su formación educacional y cultura política, los inmigrantes se integraron rápidamente a la sociedad de origen y terminaron dirigiendo las primeras organizaciones obreras, lo que demuestra lo receptivas que fueron las sociedades latinoamericanas y la necesidad de los inmigrantes de integrarse a la sociedad de acogida. ${ }^{4}$ Ahora, por esta disposición a establecer lazos con otros extranjeros y su par-

${ }^{4}$ Por ejemplo, es conocido el rol que tuvieron los inmigrantes españoles e italianos en el desarrollo del anarquismo argentino, como el famoso anarquista Enrico Malatesta. Fernando Devoto, Historia de los italianos en la Argentina, Buenos Aires, Editorial Biblos, 2008; Gonzalo Zaragoza, Anarquismo argentino, 1876-1902, Madrid, Ediciones de La Torre, 1996. 
ticipación creciente en las organizaciones nacionales de trabajadores, los estados eran conscientes de la necesidad de lograr la integración pacífica del elemento extranjero. Un elemento concreto de esta política social fue el Hotel del Inmigrante, instancia de acogida del extranjero que fue conocida en Brasil y Argentina. Los inmigrantes eran recibidos, clasificados e integrados en un programa que incluía el aprovisionamiento de alimentación, salud y trabajo. Los gobiernos mostraron con orgullo esta institución como la prueba de que estaban dispuestos a resolver los problemas sociales que la inmigración provocaba.

La asociación entre la inmigración y el anarquismo fue transversal a las elites latinoamericanas que vieron en la llegada masiva de extranjeros una variable peligrosa de la "cuestión social": la dimensión internacional del problema. Para enfrentarlo ya no sería suficiente dar cuenta de las condiciones de vida de la población trabajadora o de los procesos de urbanización e industrialización nacional, sino también de las ideas extranjeras que arribaban sin control y de la circulación del factor trabajo. La respuesta más habitual fue echar mano al aparato policial y jurídico del Estado, dictando leyes de residencia o de defensa social. Estas leyes ayudaron a criminalizar la figura del "inmigrante intelectual" que se diferenciaba del inmigrante tradicional por su labor destacada en los círculos obreros urbanos, por su participación en los medios intelectuales en formación y cuya posición, con respecto a la sociedad de acogida, era de crítica constante y acción subversiva.

Sin embargo, las leyes de residencia, con todo lo efectivas que pudieron ser al expulsar al inmigrante subversivo, dejaron en pie el problema ya que los extranjeros trasladaban su actividad política fuera de las fronteras, manteniendo los contactos con sus antiguos compañeros. Esto fue importante en las áreas fronterizas que por su integración cultural o condiciones particulares favorecieron la mantención de los contactos: Uruguay, Argentina y Chile o México y Estados Unidos.

En este objetivo de desarticular las redes subversivas es que los gobiernos comenzaron a colaborar entre ellos en la lucha en contra de los anarquistas. Que la Segunda Conferencia Panamericana 
avanzara en la coordinación de esa lucha se explica no sólo por el temor creciente a la presencia de ideologías de disolución social en los distintos países, ni por la constatación de la existencia de redes transnacionales anarquistas, sino por los tempranos cambios que algunos países (en especial Estados Unidos) buscaron darle a la Unión de Repúblicas Americanas. Ya la Primera Conferencia había resuelto que los países americanos avanzarán en un derecho penal americano, con vista a la integración de sus códigos penales.

Este tratado de extradición que presentamos merece una atención especial en al menos dos aspectos. En primer lugar, este esfuerzo coordinador entre los países del continente parece ser el primero en su tipo y responde a la necesidad de enfrentar con criterios supranacionales un problema que se resistía a ser pensado al interior de las fronteras de un país. Considerando que los problemas asociados al anarquismo eran desiguales entre las naciones, el éxito del tratado dependería del esfuerzo de sensibilización hacia los gobiernos que veían como lejana la problemática. En cierta forma su existencia y el compromiso de las naciones a respetarlo obligaban a los estados a incorporar, en sus agendas políticas nacionales, la cuestión del anarquismo. El que algunos países pusieran objeciones a extraditar detenidos, que bajo la legislación de los países requerientes significara pena de muerte, mostraba la necesaria independencia con que los gobiernos participaban del mismo y de las distintas perspectivas con que veían el problema.

En segundo lugar, este tratado de extradición buscaba la coordinación de los esfuerzos en la lucha contra el anarquismo, obligando recíprocamente a todos los países a negar el refugio a los sujetos que cometieran delitos considerados en el tratado. Si bien existían antecedentes de convenios de colaboración en el continente éstos nunca hicieron referencia al anarquismo y no se enmarcaron en la política de asistencia regional propia del panamericanismo. ${ }^{5}$

El objetivo del tratado de criminalizar el anarquismo queda en evidencia al seńalar de manera sistemática y amplia los actos delicti-

${ }^{5}$ Nos referimos al Tratado de Extradición del Congreso Americano de Jurisconsultos de Lima en 1879 y al Tratado sobre derecho procesal penal internacional, firmado en Montevideo el 23 de enero de 1889 en el Primer Congreso Sudamericano de Derecho Internacional Privado. 
vos factibles de formar parte del proceso de extradición. Si en una primera lectura el conjunto de delitos se enmarca en lo que contemporáneamente se conoce como delitos comunes, con el tratado se buscaba instalar en el imaginario penal del continente la imagen del anarquista como delincuente. La amenaza del anarquismo aparecía configurada como violencia común, desprendida de toda ideología y fundamento político. Al desconocer la dimensión política del anarquismo se desconocía su derecho de asilo. De paso, y frente a la imposibilidad de distinguir entre el "verdadero" delincuente y el anarquista, el tratado evitaba que los primeros se escudaran en falsas posiciones políticas para la condonación de sus penas o granjearse el favor de la opinión pública. Esto explica que el tratado, en su artículo núm. 2, excluyera del proceso de extradición los delitos que el mismo tratado calificaba como políticos. Con ello se garantizaba el derecho de asilo a todos los políticos que quisieran participar en proyectos de desestabilización de los regímenes de turno, derecho negado por el mismo artículo a los anarquistas.

En fin, lo que no midieron los redactores y firmantes del Tratado es que ayudaron a diluir aún más los límites entre delincuencia y lucha política, alimentando el aura de salteador, bandolero, malhechor y forajido asignado a los anarquistas, pero al mismo tiempo el de consecuente, perseguido, proscrito y expatriado. Con esta estigmatización quizás se reduciría el número de sus militantes, pero posiblemente también se aumentaría el número de sus seguidores.

\section{DOCUMENTO}

\section{TrATAdo}

Art. 1. Las altas Partes Contratantes convienen en entregarse recíprocamente a las personas acusadas o sentenciadas por autoridad competente, siempre que concurran las siguientes circunstancias:

I. Que el Estado requeriente tenga jurisdicción para encausar al delincuente que motive la demanda de extradición.

II. Que se invoque la perpetración de un crimen o delito del 
orden común, que las leyes de los Estados requeriente y requerido castiguen con una pena no menor de dos ańos de prisión.

III. Si, con motivo del régimen federal de alguna o algunas de las Altas Partes Contratantes, no fuere posible determinar la pena correspondiente al delito por el cual se pide la extradición, se tendrá entonces por base para la demanda, la siguiente lista de delitos:

1.- Homicidios, inclusos los delitos conocidos con los nombres de parricidio, asesinato, envenenamiento e infanticidio. 2.- Estupro y violación.

3.- Bigamia.

4.- Incendio.

5.- Crímenes o delitos cometidos en el mar; a saber: A) Piratería, según se conoce y se define comúnmente en Derecho Internacional. B) Destrucción o pérdida de un buque, causadas intencionalmente, o conspiración y tentativa para conseguir dicha destrucción o pérdida, cuando hubieren sido cometidas por alguna persona o personas a bordo de dicho buque en alta mar. C) Motín o conspiración por dos o más individuos de la tripulación, o por otras personas a bordo de un buque de alta mar, con el propósito de rebelarse contra la autoridad del Capitán o Comandante de dicho buque, o con el de apoderarse por fraude o violencia de dicho barco.

6.- Allanamiento de morada, por el cual se entenderá el acto de asaltar la casa de otro y de entrar en ella durante la noche, con el fin de cometer un delito.

7.- El acto de forzar la entrada a las oficinas públicas, Bancos, Casa de Banco, Cajas de Ahorro, Compañías de Depósito o de Seguros, con el fin de cometer en ellas un robo, así como los robos que resulten de ese acto.

8.- Robo con violencia, entendiéndose por tal, la substracción por la fuerza de bienes o dinero ajenos, o ejerciendo violencia o intimidación.

9.- Falsificación o expendio, o circulación de documentos falsificados. 
10.-Falsificación o alteración de los actos oficiales del Gobierno de la autoridad pública, inclusos los tribunales, o el empleo o uso fraudulento de algunos de los mismos actos. 11.- Falsificación de moneda, sea en metálico o en papel, de títulos o cupones de deuda pública, u otros títulos de crédito público, de billetes de Banco, de sellos, timbres, cuños y marcas de la nación o de la administración pública, y el expendio, circulación o uso fraudulento de alguno de los objetos antes mencionados.

12.- Importación de instrumentos para falsificar moneda, o billetes de Banco, o papel moneda.

13.- Peculado o malversación de fondos públicos, cometidos dentro de la jurisdicción de cualquiera de las Partes Contratantes, por empleados o depositarios públicos.

14.- Abuso de confianza cometido con fondos de un Banco de Depósito o de una Caja de Ahorros, o de una Compañía de Depósito, organizada conforme a las leyes.

15.- Abuso de confianza por una persona o personas a sueldo o salario, en perjuicio de aquel que los tiene a su servicio, cuando el delito está sujeto a una pena conforme a las leyes del lugar donde fue cometido.

16.- Plagio de menores o adultos, entendiéndose por tal el hecho de apoderarse de una persona o personas, o detenerlas para exigir dinero por su rescate o para cualquier otro fin ilegal.

17.- Mutilación o inutilización de cualquier miembro principal del cuerpo, y cualquiera otra mutilación intencional que cause incapacidad para trabajar, o la muerte.

18.- Destrucción maliciosa o ilegal, o la tentativa de destrucción de ferrocarriles, trenes, puentes, vehículos, buques u otros medios de comunicación, o de edificios públicos o privados, cuando el acto cometido ponga en peligro la vida human.

19.- Obtener por medio de amenazas de hacer daño, o de maquinaciones o artificios, dinero, valores $\mathrm{u}$ otros bienes muebles, o la compra de los mismos a sabiendas de cómo se 
han obtenido, cuando estos delitos estén penados con prisión u otro castigo corporal por las leyes de ambos países. 20.- Hurto o robo sin violencia, entendiéndose por tal el apoderamiento de efectos, bienes muebles, caballos, ganado vacuno o de otra clase, o de dinero, por valor al menos de veinticinco pesos, o recibir a sabiendas propiedades substraídas de ese valor.

21.- El conato de algunos de los delitos antes enumerados, cuando esté penado con prisión u otra pena corporal por las leyes de ambas Partes Contratantes.

IV. Que el Estado requeriente presente documentos que, según sus leyes, autoricen la prisión preventiva y el enjuiciamiento del reo.

V. Que el delito o la pena no estén prescritos, según las leyes de ambos países.

VI. Que el reo, si ha sido sentenciado, no haya cumplido su condena.

Art. 2. No podrá concederse la extradición por delitos políticos o por hechos que les sean conexos. No serán reputados delitos políticos los actos que estén calificados de anarquismo por la legislación del país requeriente y por la del requerido.

Art. 3. En ningún caso la nacionalidad de la persona acusada podrá impedir su entrega en las condiciones estipuladas por el presente Tratado; pero ningún Gobierno estará obligado a conceder la extradición de sus propios ciudadanos, sino que podrá entregarlos cuando a su juicio sea conveniente hacerlo.

Art. 4. Si la persona cuya extradición se solicita se encuentra sujeta a un procedimiento penal, o está detenida por haber delinquido en el país donde se ha refugiado, deberá diferirse su entrega hasta la conclusión del proceso, o hasta que haya cumplido su condena.

Art. 5. La extradición acordada no autoriza el enjuiciamiento y castigo del individuo entregado, por delito distinto del que hubiese 
servido de fundamento a la demanda respectiva, a no ser que tenga conexión con el que la motivó y se funde en las mismas pruebas de la demanda.

Esta estipulación no se aplica a los crímenes o delitos cometidos con posterioridad a la extradición.

Art. 6. Si otro u otros Estados, en virtud de estipulaciones de tratados, solicitan la entrega de un mismo individuo por motivo de diferentes delitos, se atenderá, en primer lugar, el pedido de aquel en cuyo territorio, a juicio del Estado requerido, se haya cometido la infracción más grave. Si los delitos fueran estimados de la misma gravedad, se dará preferencia al Estado que tenga prioridad en el pedido de extradición; y si todos los pedidos tuvieran la misma fecha, el país requerido determinará el orden de la entrega.

Art. 7. Las demandas de extradición serán presentadas por medio de los Agentes Diplomáticos o Consulares respectivos; y a falta de éstos, directamente de Gobierno a Gobierno; e irán acompañadas de los siguientes documentos:

I. Respecto de los presuntos delincuentes: copia legalizada de la ley penal aplicable a la infracción que motivare la demanda, y del auto de prisión y demás documentos a que se refiere la fracción IV del artículo $1^{\circ}$.

II. Respecto de los sentenciados: copia legalizada de la sentencia condenatoria ejecutoriada.

Deberán también acompañarse a la demanda todos los datos y antecedentes necesarios para establecer la identidad de la persona cuya extradición se reclamare.

Art. 8. En caso de urgencia, se podrá conceder la detención provisional del individuo reclamado, en virtud de petición telegráfica del Gobierno requeriente al Ministerio de Relaciones Exteriores, o a la autoridad competente del requerido, en la cual se prometa el envío de los documentos indicados en el artículo anterior; pero el detenido será puesto en libertad, si éstos no fueren presentados dentro del 
término que fije la nación requerida, no excediendo de tres meses, contados desde la fecha del arresto.

Art. 9. La demanda de extradición, en cuanto a sus trámites, a la apreciación de la legitimidad de su procedencia, y a la admisión y calificación de las excepciones con que pudiese ser impugnada por parte del reo o prófugo reclamado, quedará sujeta, en lo que no se oponga a lo prescrito en este Tratado, a la decisión de las autoridades competentes del país de refugio, las cuales arreglarán sus procedimientos a las disposiciones y prácticas legales establecidas para el caso en el mismo país. Queda garantizado al reo prófugo el derecho de usar el recurso de Habeas Corpus o amparo de sus garantías individuales.

Art. 10. Todos los objetos que se encontrasen en poder del acusado, si los hubiere obtenido por medio de la perpetración del hecho del que se le acusa, o pudiesen servir de prueba del delito por el cual se pide su extradición, serán secuestrados y entregados con su persona. Sin embargo, quedarán a salvo los derechos de terceros sobre las cosas secuestradas, si no estuviesen implicadas en la acusación.

Art. 11. El tránsito por el territorio de uno de los Estados Contratantes, de algún individuo entregado por tercera Potencia a otro Estado y que no pertenezca al país de tránsito, será concedido mediante simple presentación, en original o en copia legalizada de la resolución, en que se haya concedido la extradición por el Gobierno del país de refugio.

Art. 12. Todos los gastos ocasionados con la extradición de un prófugo serán a cargo del Estado requeriente, exceptuándose las compensaciones de los funcionarios públicos que reciban sueldos fijos.

Art. 13. La extradición de todo individuo culpable de actos de anarquismo puede pedirse siempre que las legislación de los Estados, requeriente y requerido, haya establecido la pena para dichos actos. En este caso, la extradición de concederá aun cuando el delito imputado al reclamado tuviere una pena menor de dos ańos de prisión. 
Art. 14. Los Gobiernos Contratantes convienen en sujetar a arbitraje las controversias que puedan suscitarse acerca de la interpretación o ejecución de este Tratado, cuando se hayan agotado los medios de arreglo directo.

Cada Parte Contratante nombrará un árbitro, y los dos árbitros designarán un tercero para el caso de discordia. La Comisión de Árbitros determinará el procedimiento arbitral en cada caso.

Art. 15. El presente Tratado permanecerá en vigor durante cinco años, contados desde el día en se haga el ultimo canje de ratificaciones, y seguirá en vigor por otros cinco años más, si doce meses antes de que expire el primer periodo de cinco ańos no fuere denunciado. En el caso de que alguno o algunos de los Gobiernos lo denunciare, seguirá en vigor entre las otras Partes Contratantes. Esta Convención será ratificada y las ratificaciones se canjearán en la Ciudad de México, dentro del término de un año de su firma.

Art. 16. Si algunas de la Altas Partes Contratantes hubieren celebrado ya entre sí tratados de extradición, quedarán éstos reformados solamente en la parte modificada o alterada por las disposiciones del presente.

\section{ARTíCUlo TRANSITORIO}

Los representantes de Costa Rica, Ecuador, Honduras y Nicaragua firmaran este Tratado con la reserva de que sus respectivos Gobiernos no entregarán a los delincuentes que merezcan pena de muerte, según la legislación de los países requerientes, sino bajo la promesa de que se les conmutará esa pena por la inmediata inferior.

Si los gobiernos de las Delegaciones mencionadas mantienen la misma reserva al ratificar el presente Tratado, éste los ligará únicamente con aquellos que acepten la mencionada condición.

En de de lo cual los Plenipotenciarios y Delegados firman el presente Tratado y ponen en él el sello de la Segunda Conferencia Internacional Americana. Hecho en la Ciudad de México el día veintiocho de Enero de mil novecientos dos, en tres ejemplares es- 
critos en castellano, inglés y francés respectivamente, los cuales se depositarán en la Secretaría de Relaciones Exteriores del Gobierno de Estados Unidos Mexicanos, a fin de que de ellos se saquen copias certificadas para enviarlas por la vía diplomática a cada uno de los Estados signatarios.

Países firmantes: Argentina, Bolivia, Colombia, Costa Rica, Chile, República Dominicana, Ecuador, El salvador, Estados Unidos, Guatemala, Haití, Honduras, México, Nicaragua, Paraguay, Perú y Uruguay. 